\title{
The Distribution of Reaction Rates and Activation Energies on Catalytic Surfaces: Exchange Reaction between Gaseous Benzene and Benzene Adsorbed on Platinum
}

\author{
J. A. BRUNDEGE* AND G. PARRAVANO \\ From the Department of Chemical and Metallurgical Engineering, University of Michigan, \\ Ann Arbor, Michigun
}

\begin{abstract}
A mathematical derivation for the calculation of the distribution function of rate constants and activation energies for an isotopic exchange reaction between chemical species adsorbed on a solid surface and in the surrounding gas phase is presented. Starting from experimental kinetic isotherms, giving the rate of exchange as a function of time at various temperatures, it is shown how it is possible to derive the distribution functions of the rate constants and activation energies. The technique is applied to the exchange reaction between benzene molecules adsorbed on Pt black and molecules present in the gas phase. Experimental results on the rate of this exchange, obtained by means of C-14 labeled benzene, have been collected at $40^{\circ}, 60^{\circ}, 80^{\circ}$, and $100^{\circ} \mathrm{C}$, at constant surface coverage. The range of activation energies for the exchange reaction was found to be from about 10 to $35 \mathrm{kcal} / \mathrm{mole}$, while the pre-exponential factor varied from about $10^{7}$ to $10^{26} \mathrm{~min}^{-1}$. Additional observations on the chemisorption of benzene on $\mathrm{Pt}$ bluck are presented. The effects of various pretreatments of the Pt surface and of aging of the chemisorbed benzene on the exchange rate are discussed.
\end{abstract}

\section{INTRODUCTTON}

Extensive studies have been carried out in the past on the nature of the energy distribution at catalytic surfaces, and the interest of the investigators has been particularly attracted by those surfaces which have clearly shown a nonuniform $f$ localization of the energy. The deviations of adsorption isotherms and isobars of many solid-gas systems from the "ideal" Langmuir behavior, the variations of adsorption heat effects with the amount of surface covered, and the results on adsorption kinetics have served as the experimental basis for theoretical deductions on chemisorption processes at nonuniform surfaces (1). In general the measurements underlying these methods suffer from the fact that the possible influence of interactions among adsorbate mole-

\footnotetext{
* Present Address: Monsanto Chemical Company, St. Louis, Missouri.

$\dagger$ In this communication, the terms nonuniform and heterogeneous when applied to a solid surface are considered synonyms.
}

cules is superimposed on the effect of the nonuniform distribution of the energy on the surface of the adsorbent. Since interaction phenomena are dependent upon the degree of surface coverage, observations on surface heterogeneity are best carried out at constant surface coverage. In this direction the use of isotopic molecules has proved to be an invaluable tool to establish the heterogeneous nature of several solid surfaces. In the kinetic isotopic method (3), which involves measurements on the rate of isotopic exchange reactions between adsorbed and gas phase species, each labeled molecule leaving the surface is replaced by a chemically identical molecule. Thus the surface coverage, and hence the interactions among adsorbed molecules remain constant.

This method has already been used to gather information on catalytic surfaces including the adsorption of $\mathrm{CO}$ on $\mathrm{Fe}(2)$, of $\mathrm{H}_{2}$ on $\mathrm{Ni}-\mathrm{SiO}_{2}(4)$, of $\mathrm{CO}$ on $\mathrm{Ni}$ films $(5)$, and of $\mathrm{H}_{2}$ on $\mathrm{Pt}(6)$. In the first three studies cited the method was used only to demonstrate qualitatively the heterogeneity of the 
surface, while in the latter instance, the relation between the activation energy of the $\mathrm{H}_{2}-\mathrm{D}_{2}$ reaction and the extent of conversion was obtained by comparing the rate of reaction at different temperatures for the same extent of conversion. This procedure implies that molecules adsorbed on surface regions, corresponding to a value of the activation energy of reaction lower than a fixed quantity, will all have reacted, while the opposite will occur to molecules adsorbed on surface sites possessing a higher value of the reaction activation energy. This, of course, is not strictly valid, since adsorbed species from all regions of the surface contribute to the observed rate. The activation energy of the exchange reaction cannot be shown as a function of the fraction reacted, but can be represented as a function of the fraction of surface sites having activation energies less than or equal to a certain value.

In the present study the kinetic isotopic method was applied to the exchange reaction:

$$
\mathrm{C}_{6}{ }^{*} \mathrm{H}_{6}(\mathrm{ads})+\mathrm{C}_{6} \mathrm{H}_{6}(\mathrm{~g}) \rightarrow \mathrm{C}_{6} * \mathrm{H}_{6}(\mathrm{~g})+\mathrm{C}_{6} \mathrm{H}_{8}(\mathrm{ads})
$$

where $\mathrm{C}_{6} \mathrm{H}_{6}(\mathrm{ads})$ and $\mathrm{C}_{6} \mathrm{H}_{6}(\mathrm{~g})$ represent a benzene molecule adsorbed on a solid surface and one in the gas phase, respectively. Pt was chosen as the adsorbent. The adsorption of benzene on $\mathrm{Pt}$ and other transition metals is known to occur with varying degrees of bonding strength and involving different numbers of surface atoms per molecule adsorbed $(\gamma, 8)$. It was hoped that this situation would be of interest in discussions on the nature of the energy distribution at solid surfaces. A molecular orbital theory calculation of the heat of adsorption of benzene on platinum has given the value of $108 \mathrm{kcal} /$ mole $(10)$, thus pointing to large irreversible adsorption effects.

These effects have been confirmed by the present study. We have found that benzene was adsorbed on platinum black at first very rapidly at temperatures up to $150^{\circ} \mathrm{C}$, even at pressures below $0.1 \mathrm{~mm} \mathrm{Hg}$. This was followed by a slow disappearance of gas phase benzene which, however, did not have appreciable effect on the reactivity or amount of the initially adsorbed benzene. On supported $\mathrm{Pt}$ there is a similar trend (8), and a strong irreversible adsorption of benzene corresponding to 0.22 molecules of benzene/atom of $\mathrm{Ft}$ has been reported to take place at temperatures up to $250^{\circ} \mathrm{C}$. It is also known that the adsorption of benzene on $\mathrm{Pt}$ at $83^{\circ} \mathrm{K}$ lowers the work function of the metal, indicating that $d$ electrons are displaced to the metal surface (11). Further evidence of this effect was gathered by measuring the changes in electrical conductivity of $\mathrm{Pt}$ upon chemisorption of benzene (12).

\section{Derivation of the Exchange Kinetics on a Heterogeneous Surface}

In this work the heterogeneity of a solid surface is described by the distribution of activation energies for the exchange reaction between gaseous and chemisorbed molecules. The distribution, $\rho(E)$, is defined in such a manner that $\rho(E) d E$ is the fraction of adsorption sites having an activation energy between $E$ and $E+d E$. It is assumed that for each value of the activation energy, $E$, there exists at any given temperature a corresponding value of the exchange reaction rate constant, $k$.

$$
k=k_{0} e^{-E / R T}
$$

This assumption is correct if $k_{0}$ is either a constant or a function of $T$ and $E$ only:

$$
k_{0}=f(E, T)
$$

$k_{0}$ is assumed to be a constant by most investigators. In this instance Eq. (2) becomes the Arrhenius equation. Since $k$ is a function of $E$ and $T$, at a given temperature there must exist a corresponding rate constant distribution function, $\rho(k)$, related to $\rho(E)$ by

$$
\rho(k) d k=-\rho(E) d E
$$

The minus sign in the above equation results from the fact that the activation energy and the rate constant are antibatically related.

Let us now consider an isotopic exchange reaction between gaseous molecules and molecules chemisorbed on a uniform surface (or a uniform portion of a nonuniform surface). Let the initially chemisorbed molecules be labeled and let the conditions of the reaction be such that the concentration of labeled molecules in the gas phase is negligible at all times. This condition is easily achieved in a flow-type reactor in which the 
gas containing the unlabeled molecules makes a single pass through the reactor. Under these conditions the exchange reaction must be first order and the reaction rate of the labeled molecules is proportional to the fraction of labeled molecules still chemisorbed on the surface, $\theta$ :

$$
r(t)=-d \theta / d t=k \theta
$$

Integration of the above equation from time zero to time $t$ and substitution gives:

$$
r(l)=k \mathrm{e}^{-k t}
$$

On a nonuniform surface Eq. (5) applies only to a differential portion of the surface:

$$
d r(t)=k e^{-k t} \rho(k) d k
$$

and the reaction rate for the entire surface is obtained by integrating Eq. (6) over all values of the reaction rate constant:

$$
r(t)=\int_{k_{\operatorname{mix}}}^{k_{\max }} k \rho(k) \mathrm{e}^{-k t} d k
$$

The minimum rate constant may be taken as zero, while the maximum should correspond to an activation energy of zero so that $k_{\max }=k_{0}$. Since $k_{0}$ is known to be very large, the upper limit may be taken as infinity with negligible error:

$$
r(t)=\int_{0}^{\infty} k \rho(k) \mathrm{e}^{-k t} d k
$$

Equation (8) gives an expression for the kinetic isotherm for a given rate constant distribution. In this work the reaction rates were measured as a function of time and the problem is to solve Fq. (8) for the distribution function $\rho(k)$. It may be seen that Eq. (8) is the Laplace transform of $k \rho(k)$ so that

$$
\rho(k)=(1 / k) \mathcal{L}^{-1}\{r(t)\}
$$

In order to compute $\rho(k)$ it is necessary to express the rate as a function of time in a mathematical form whose inverse transform can be computed. Since there exists no adequate theory of nonuniform surfaces the rate data must be expressed in a mathematical form which is necessarily arbitrary. It can be shown (13) that the inverse Laplace transform of a function, $r(t)$, is unique. Therefore, a given kinetic isotherm can be the result of only one rate constant distribution.
In order to obtain the distribution of the activation energy, $\rho(E)$, the relation (3) may be written

$$
\rho(E)=-\rho(k) d k / d E
$$

and from the relation (1)

$$
\frac{d k}{d E}=k\left[\frac{-1}{R T}+\frac{d\left(\ln k_{0}\right)}{d E}\right]
$$

so that

$$
\rho(E)=k \rho(k)(1 / R T-\beta)
$$

where $\beta=d\left(\ln k_{0}\right) / d E$.

If $k_{0}$ were the same for all surface sites then $\beta$ would be zero and the calculation of $\rho(E)$ would be greatly simplified. This procedure has been followed in the past $(1,14)$. It will be shown later that such an assumption is not justified, at least in the case of reaction (1) on $\mathrm{Pt}$. If the valucs of $\beta$ were known, one could calculate from Eq. (12) the values of the function $\rho(E)$ corresponding to each value of $k$; but there would still remain the problem of finding the corresponding values of $E$. In order to obtain the value of $E$ for a nonuniform surface it is necessary to compare the rate constants of similar portions of the surface at various temperatures. Under the assumption contained in $\mathrm{Eq}$. (2) the relation between adsorption sites and their rate constants (and also their activation energies) remains unchanged during variations in temperature. The relative positions of the adsorption sites with respect to ascending values of their activation energies (or descending values of their rate constants) are given by the integral distribution function, $S(E)$, which is defined by:

$$
S(E) \equiv \int_{0}^{E} \rho\left(E^{\prime}\right) d E^{\prime}
$$

where $E^{\prime}$ is a dummy variable used in place of $E$. This function may also be expressed in terms of $k$ as:

$$
S(E)=\int_{k}^{\infty} \rho\left(k^{\prime}\right) d k^{\prime}
$$

where $k$ is the rate constant corresponding to $E$. Figure 1 illustrates the relationship of the integral distribution function to the rate constants at different temperatures and 

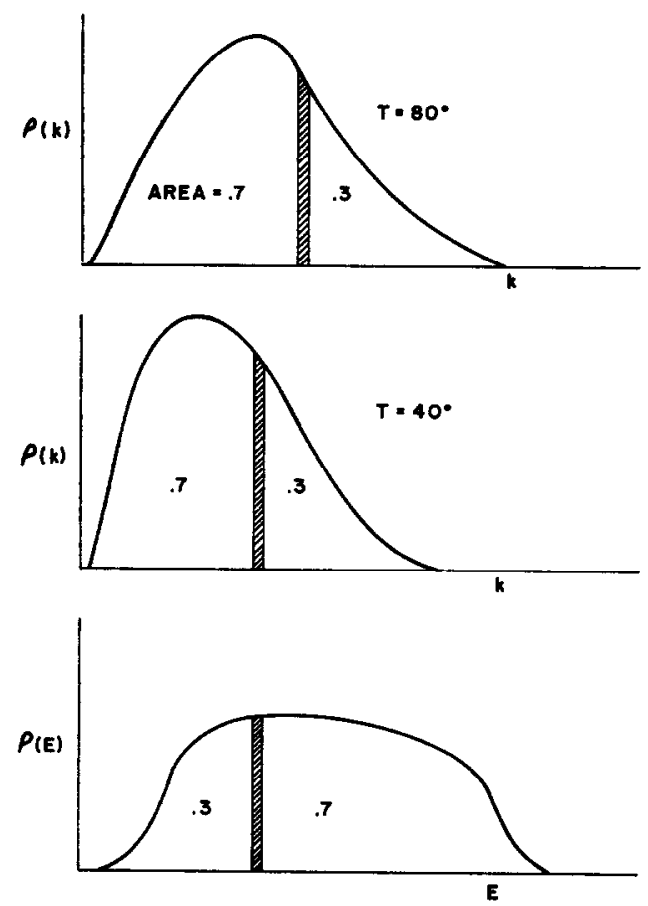

FIG. 1. An illustration showing the relationship of $\rho(k)$ and $\rho(E)$ with the integral distribution function, $S(E)$. In this example $S(E)=0.3$ which is the area under the designated portion of the distribution functions. The shaded portion under each curve refers to the same adsorption sites.

to the activation energies. The activation energy, $E$, is easily found for each value of $S(E)$. One needs only to plot $\ln k$ versus $1 / T$ at constant $S$. The slope of the plot is $-E / R$ corresponding to $S$. Similarly, a plot of $T \ln k$ versus $T$ at constant $S$ will have a slope of $\ln k_{0}$ so that the corresponding value of $k_{0}$ may also be determined. By making such plots for a wide range of values of $S(E)$, the value of $E$ is obtained as a function of $k_{0}$. The relationship of $E$ and $k_{0}$ permits one to calculate the value of $\beta$ and, consequently from Eq. (12), the value of $\rho(E)$.

\section{EXPERIMENTAL}

\section{Materials}

Thiophene-free benzene was reagent grade; helium $(99.9 \%)$ and hydrogen $(99.5 \%)$ from commercial cylinders were used without further purification. Platinum black was prepared by reduction of a solu- tion of chloroplatinic acid with formaldehyde at $5^{\circ} \mathrm{C}$. The platinum precipitate was separated from the solution by centrifugation, and washed six times with about $200 \mathrm{ml}$ of dilute $\mathrm{HCl}$ and separated by centrifugation. The platinum was dried on a steam bath, again washed six times with distilled water, and finally dried on the steam bath. The BET surface area of the platinum was found to be $9.47 \mathrm{~m}^{2} / \mathrm{g}$. Prior to the nitrogen adsorption the surface was degassed for $24 \mathrm{hr}$ at $220^{\circ} \mathrm{C}$. The effect of the degassing temperature had been investigated previously using platinum black prepared in an identical way. It was found that maintaining the platinum black at $250^{\circ}$ for several hours had no effect on the surface area; however, maintaining it at $297^{\circ}$ for $14 \mathrm{hr}$ reduced the specific surface area of one sample from 10.3 to $2.6 \mathrm{~m}^{2} / \mathrm{g}$. The platinum black was finally screened through a 200-mesh screen to break up the lumps. The reactor was charged with this powder dispersed on glass wool (26.08 $\mathrm{g}$ of Pt with $9 \mathrm{~g}$ of glass wool).

\section{Equipment}

A diagram of the equipment is shown in Fig. 2. Known amounts of labeled benzene with a specific radioactivity of about 0.003 curies/mole were introduced into the reactor by means of a burette kept at constant temperature. The quantity of labeled benzene was usually measured at a pressure of 5 to $10 \mathrm{~mm}$. At higher pressures physical adsorption on the glass walls of the burette and tubing occurred.

The reactor was kept at constant temperature $\left( \pm 0.1^{\circ} \mathrm{C}\right)$ by means of an oil bath. A by-pass around the reactor was provided in order that the flow conditions of the gas could be precisely adjusted before flow through the reactor was begun.

By means of critical flow orifices provision was made for a precisely controlled flow of either pure helium or a helium-benzene mixture containing from 1.5 to 4.5 mole $\%$ benzene. Frequent checks of the flow rate were made during the course of a run by use of a soap film flow meter. A benzenehelium mixture of known composition was obtained by bubbling helium through a series of two benzene saturators. The second 


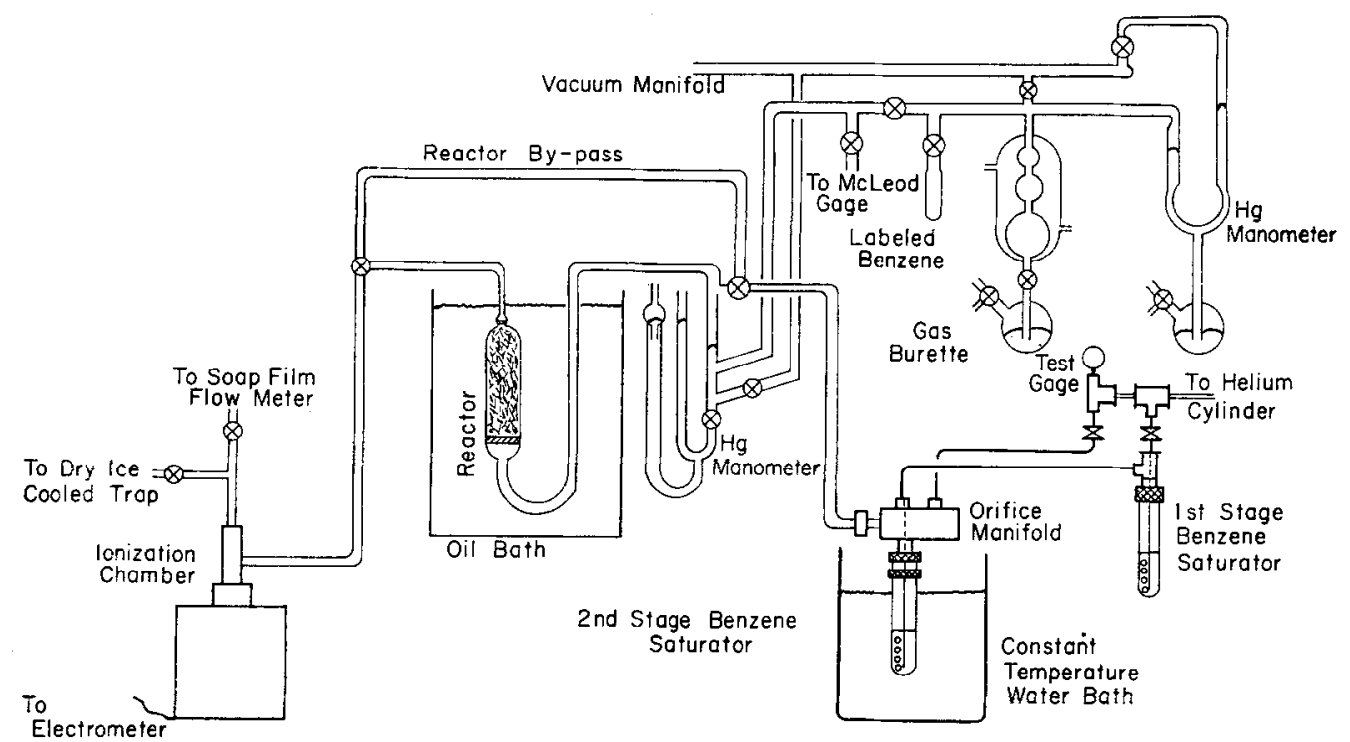

Fig. 2. Diagram of the experimental setup used to study the kinetics of reaction (1).

stage saturator was kept at $23.7^{\circ} \mathrm{C}$ in a constant temperature water bath. With a helium pressure of 97 psig, which was used most frequently, a mixture containing 1.57 mole \% benzene and a flow rate of 88.5 $\mathrm{ml} / \mathrm{min}$ were obtained.

The specific radioactivity of the gas leaving the reactor was measured with a flowtype ionization chamber. The current produced by the C-14 disintegration was measured with a Cary, Model 31, vibrating reed electrometer. A few measurements were made using the high resistance leak method in which the electrometer measured the voltage drop across a $10^{12}$ ohm resistor. However, it was found that the most reliable results could be obtained by the rate of charge method in which the ionization current is determined by observing the rate of increase of potential across the electrometer. The potential measured by the electrometer was continuously fed to a recording potentiometer. Since at a constant flow rate the amount of charge accumulating in a given time is proportional to the amount of radiocarbon that has passed through the ionization chamber during that time, the data obtained corresponded to the integral of the rate over various time intervals. From 15 to 20 time intervals were used in most of the runs.
The ionization chamber consisted of a specially constructed stainless steel shell having a volume of $40 \mathrm{ml}$. The shell was insulated from the guard ring and base by a $3 \mathrm{~mm}$ thickness of machined Teflon. The center probe, center probe insulator, guard ring, and base were taken from a $275 \mathrm{ml}$ Cary-'Tolbert flow chamber (Applied Physics Corp.). The use of the Cary-Tolbert chamber was first attempted and found to be unsatisfactory because of appreciable adsorption of benzene by the rubber insulators of the chamber.

Calibration of the ionization chamber and obscrvations on the transient response of it and the empty reactor were made using a reactor similar to the one used in the experiments but having a smaller volume. The current produced in the ionization chamber was somewhat sensitive to the flow rate, giving a lower current at a higher flow rate. In addition, a sudden change in the flow rate through the ionization chamber would cause a false reading which persisted for 5 to $10 \mathrm{~min}$.

During the calibration a known quantity of tagged benzene was introduced into the reactor. Helium was then admitted to the reactor to bring the total pressure up to 1 atm. The total amount of charge produced in the electrometer as the tagged benzene 
was swept out of the reactor and through the ionization chamber was measured. For a flow rate of $140 \mathrm{ml} / \mathrm{min}$ the calibration factor was $3.06 \times 10^{-4} \mu \mathrm{moles} /(\mathrm{ml} / \mathrm{min}) /(\%$ of $30 \mathrm{mv}$ scale), while for a flow rate of 87.3 $\mathrm{ml} / \mathrm{min}$ the calibration factor was $2.94 \times$ $10^{-4}$

When pure helium was used to sweep the tagged benzene out of the reactor about $85 \%$ would be swept out during the first $30 \mathrm{~min}$ and about $99 \%$ would be swept out in $4 \mathrm{hr}$. When a helium-benzene mixture containing about $1.5 \%$ benzene was used to sweep the tagged benzene out of the reactor, $99 \%$ of the tagged benzene would be swept out during the first $60 \mathrm{~min}$.

\section{Procedure}

Prior to each run the platinum surface was cleaned, reactivated, and covered with chemisorbed labeled benzene by the following procedure:

1. The reactor was purged with hydrogen for $1 \mathrm{hr}$ at a temperature of $120^{\circ}$. This treatment removed all of the previous benzene from the platinum surface.

2. Subsequently the reactor was evacuated for about $1 / 2 \mathrm{hr}$; air was admitted slowly and flowed through the reactor for a few seconds. This treatment oxidized the chemisorbed hydrogen and reactivated the surface. Elimination of this step resulted in a surface with a greatly reduced adsorptive capacity.

3. Then the reactor was evacuated for about 1/2 hour in order to remove all of the gas phase oxygen. Then hydrogen was admitted slowly to remove chemisorbed oxygen.

4. Next the reactor was evacuated, heated to $150^{\circ}$, and kept under vacuum at $150^{\circ}$ for $20 \mathrm{hr}$. At the end of the $20 \mathrm{hr}$ period the reactor pressure was about $10^{-6} \mathrm{~mm}$. This treatment removed most of the adsorbed hydrogen.

5. The reactor was cooled to $120^{\circ}$ and labeled benzene vapor was admitted to the reactor in one or more measured portions. Usually benzene would be admitted until a sharp rise in pressure was observed. It was found that an "equilibrium" pressure of $0.05 \mathrm{~mm}$ represented about a $10 \%$ excess of benzene over that required to completely fill the strong adsorption sites. In general, this corresponded to a surface coverage of the $\mathrm{Pt}$ of about 0.09 , varying between 0.11 at the beginning of the series of runs and 0.06 at the end.

6. Helium was admitted to the reactor to bring it to atmospheric pressure and helium flowed through the reactor at a measured rate. The amount of labeled benzene swept out of the reactor and flowing through the ionization chamber was measured. The helium purge was continued until no labeled benzene was detectable in the reactor effluent.

7. Twenty-four hours after the labeled benzene was first admitted to the reactor, the latter was quickly cooled from $120^{\circ}$ to the desired exchange reaction temperature. Adsorption at $120^{\circ}$ and aging for $24 \mathrm{hr}$ at this temperature was the standard conditioning the system received before each run. The longer the aging period and the higher the aging temperature the slower the exchange reaction rate was found to be. By aging the chemisorbed benzene for $24 \mathrm{hr}$ at a temperature substantially above the reaction temperature, a negligible amount of aging occurred during the course of the exchange reaction, which generally lasted from 2 to 5 days. The amount of aging that the system received was the same for all runs regardless of the run temperature.

After the reactor had cooled to the desired temperature and the exchange reaction was begun, the concentration of labeled benzene in the reactor effluent was measured in the ionization chamber. After several days of measurements, the rate of the exchange reaction became exceedingly slow, and the reaction was stopped by purging all gas phase benzene from the reactor with pure helium. The reactor was then heated to a higher temperature while continuing the helium purge, the exchange reaction restarted, and the rates measured as described in the preceding paragraph. This step was repeated again at a higher temperature. The last and highest temperature used in each experiment was $120^{\circ}$.

\section{Results}

The experimental technique described above was evolved through a series of experi- 
ments on three different preparations of platinum black. Only the results obtained on the last preparation are reported here (15). Eighteen runs were made or attempted on this preparation. Seven of these were considered sufficiently accurate for use in computing the distribution function. These are summarized in Table 1. the various treatments of $\mathrm{Pt}$ black were studied by collecting and analyzing samples of the materials swept out of the reactor by the various gases flowing through it. The samples were obtained by condensation in a Dry-Ice-cooled trap. In those instances where the quantity of condensable matcrial was very small, toluene was placed in the

TABLE 1

Summary of Runs on the Exchange Reaction (1)

\begin{tabular}{|c|c|c|c|c|c|c|c|}
\hline \multirow[b]{2}{*}{$\underset{\text { number }}{\text { Run }}$} & \multirow[b]{2}{*}{$\begin{array}{c}\text { Labeled } \mathrm{C}_{6} \mathrm{H}_{6} \\
\text { introdduced } \\
\text { at } 120^{\circ} \\
(\mu \text { moles })\end{array}$} & \multirow{2}{*}{$\begin{array}{c}\text { Labeled } \mathrm{C}_{6} \mathrm{H}_{6} \\
\text { swept out by } \\
\text { helium in } 24 \mathrm{hr} \\
\text { at } 120^{\circ} \\
\text { ( } \mu \text { moles) }\end{array}$} & \multicolumn{3}{|c|}{ For exchange reaction } & \multirow{2}{*}{$\begin{array}{c}\text { Additional } \\
\text { amount } \\
\text { reacting at } \\
\text { higher temp. } \\
(\mu \text { moles })\end{array}$} & \multirow{2}{*}{$\begin{array}{l}\text { Average deviation } \\
\text { of Eq. (26) } \\
\text { from the } \\
\text { experimental rates } \\
(\%)\end{array}$} \\
\hline & & & $\underset{\left({ }^{\circ} \mathrm{C}\right)}{\text { Temp. }}$ & $\begin{array}{l}\text { Length } \\
\text { of run } \\
\text { (days) }\end{array}$ & $\begin{array}{l}\text { Amount } \\
\text { reacting } \\
\text { ( } \mu \text { moles) }\end{array}$ & & \\
\hline 7 & 135.3 & 14.6 & 80 & 2 & 92.3 & 17.8 & 2.60 \\
\hline 8 & 113.2 & 12.2 & 60 & 4 & 71.7 & 28.3 & 1.88 \\
\hline 9 & 116.8 & 13.3 & 60 & 3 & 66.3 & 25.9 & 1.26 \\
\hline 11 & 108.0 & 20.6 & 40 & 3 & 40.0 & 37.0 & 1. 32 \\
\hline 12 & 80.9 & 3.8 & 80 & 2 & 59.9 & 12.0 & 1.20 \\
\hline 15 & 72.6 & 6.9 & 40 & 3 & 33.7 & 29.7 & 1.51 \\
\hline 16 & 64.3 & 5.5 & 100 & 1 & 52.7 & 2.8 & 2.38 \\
\hline
\end{tabular}

The amount of strongly adsorbed benzene decreased with each succeeding run. The cause of this is uncertain. It may be due to sintering of the platinum or a partial poisoning of the surface. In order to compare the results from different runs it was necessary to normalize the rates by dividing the amount reacting in each time interval by the amount which ultimately reacted during that run. To determine the latter it was necessary to increase the reactor temperature to $120^{\circ}$ and continue the exchange reaction until only traces of labeled material could be found leaving the surface.

It may be seen from Table 1 that more labeled benzene is introduced into the reactor than has ultimately reacted by exchange. This difference, which ranges from 1 to $10 \%$, is probably due to decomposition of some of the adsorbed benzene on the surface. In general, the greater the excess of labeled benzene introduced into the reactor over that which is strongly adsorbed the greater the amount lost by decomposition. This is in agreement with previous work which showed a steady pressure-dependent loss of benzene when it was contacted by a supported Pt catalyst ( 8 ).

The chemical changes occurring during trap to act as an absorbent. A gas-liquid chromatography unit containing a supported tritolyl-phosphate-packed column was used to analyze the samples. With a microliter of liquid sample, concentrations as low as $200 \mathrm{ppm}$ could be detected.

Material collected during the helium sweep of the reactor, following the adsorption of labeled benzene, showed that only pure benzene was swept out by the helium. No trace of cyclohexane, methane, diphenyl, or other hydrocarbon could be detected. Material collected during the first $30 \mathrm{~min}$ of the exchange reaction, during which 20 to $50 \%$ of the labeled benzene left the surface, also showed that only pure benzene was present. No trace of other organic compounds could be detected in the reactor effluent even after a helium-benzene mixture had been kept in the reactor for several hours at $120^{\circ}$. The hydrocarbons swept out of the reactor by the hydrogen purge consisted mainly of cyclohexane plus about $2 \%$ of another hydrocarbon, probably methylcyclohexane. A trace of benzene was sometimes detected. No trace of methane or $n$-hexane was found. The material swept out of the reactor during the air purge, following the hydrogen treatment, consisted 
mostly of water vapor and possibly some carbon dioxide. No hydrocarbons were detected.

There are many conditions which must be specified in order to completely define the system of benzene chemisorbed on Pt. They are not all known. The surface pretreatment used in the runs of Table 1 consisted of exposure to hydrogen, then air, then hydrogen again, and finally degassing under vacuum for $20 \mathrm{hr}$ at $150^{\circ}$. Variations of this pretreatment gave different results but this effect was not studied quantitatively. Likewise, the temperature of chemisorption and the time and temperature of aging of the system after adsorption were found to have a large effect on the exchange reaction rate but were not studied quantitatively. One run was made by adsorbing and aging at $150^{\circ}$ instead of the usual $120^{\circ}$. The rate was about one-third as fast as during run 16 .

The concentration of ordinary benzene in the gas phase was $1.57 \%$ for the runs in Table 1 . Benzene concentrations as high as $3.5 \%$ were used in some of the early runs. The higher benzene concentrations appeared to give a slightly higher reaction rate but the increase was not significantly greater than the reproducibility of these runs.

\section{Correlation of the Experimental Results}

In order to calculate the distribution function from the reaction rate measurements the rate results must be put into some mathematical form whose inverse Laplace transform can be calculated. In addition, there are certain physical requirements which a rate equation for this type of reaction must conform to. These are

$$
\begin{gathered}
r(0)=\text { finite number } \\
\int_{0}^{\infty} r(t) d t=1.0 \\
d r / d t<0 \text { for } 0 \leqslant t<\infty \\
d^{2} r / d t^{2}>0 \text { for } 0 \leqslant t<\infty \\
r=d r / d t=d^{2} r / d t^{2}=0 \text { at } t=\infty
\end{gathered}
$$

Conditions (15), (16), and (19) are obvious. Conditions (17) and (18) arise from the fact that an isotopic exchange of the type used in this work is kinetically first order. The heterogeneity of the surface does not alter this conclusion, but may be simply thought of as producing a series of a large number of simultaneous first order reactions.

In addition to the above conditions for the rate, $\rho(k)$, which is calculated from the rate expression, must satisfy the following conditions:

$$
\begin{gathered}
\int_{0}^{\infty} \rho(k) d k=1.0 \\
\rho(k) \geqslant 0 \text { for } 0<k \infty \\
\rho(\infty)=0 \\
\rho(0)=0
\end{gathered}
$$

Condition (20) is similar to condition (15) and both result from the use of normalized rates and distributions. Conditions (21), (22), and (23) are obvious.

A frequently used empirical equation for solid-gas reactions, especially adsorption and desorption, is the Elovich equation

$$
r(t)=a /\left(t+t_{0}\right)
$$

Quite obviously, this equation fails to satisfy conditions (15) and (20) and must, therefore, be rejected. In the literature, the following equation is mentioned as a possible correlation function for desorption data (1b):

$$
q(t)=\sum_{j=1}^{N} a_{j} /\left(t+t_{0}\right)^{j}
$$

where $t_{0}$ and $a_{j}$ 's are constants and $q(t)$ is the fraction desorbed in time $t$. The corresponding rate equation is

$$
r(t)=\sum_{j=1}^{N} j a_{j} /\left(t+t_{0}\right)^{j+1}
$$

This equation meets every condition except (23), provided $t_{0}>0$. If the summation were started at $j=2$, condition (23) would also be satisfied. However, the $a_{j}$ 's would have to be positive if conditions (16) and (17) are to be satisfied at all values of $t$. In principle these requirements can be satisfied even with a few negative $a_{j}$ 's provided a sufficiently large number of terms are used. The principal practical difficulty of Eq. (25) is that, at the larger values of $j$, one 
would have to deal ${ }_{-2}^{-}$with extremely large numbers.

The equation which was finally used for the correlation of the rate data was the following:

$$
r(t)=\sum_{j=1}^{N} a_{j} /\left(t+b_{j}\right)^{3}
$$

where the $a_{j}$ 's and $b_{j}$ 's are positive constants. This is very similar to Eq. (25), and satisfies all of the conditions (15) through (23) provided that

$$
\sum_{j=1}^{N} a_{j} / 2 b_{j}^{2}=1.0
$$

The inverse Laplace transform of $(26)$ is

$\mathfrak{L}^{-1}\{r(t)\}=k \rho(k)=\left(k^{2} / 2\right) \sum_{j=1}^{N} a_{j} \mathrm{e}^{-b_{j} k}$

A good fit of the experimental data could be obtained with Eq. (26). It was necessary to preselect arbitrarily the values of $b_{j}$. The $a_{j}$ 's were then fit to the experimental data by least squares. It was found that the values arbitrarily chosen for the $b_{j}$ 's had very little effect on either the goodness of fit or the resulting distribution function, provided the chosen values covered a wide range. All of the data processing and calculations were made on an IBM 709 computer (15). The results of the data correlation were immediately used by the computer to calculate $\rho(k), k,-\ln k$, and $-T \ln k$ for a wide range of values of $S(E)$. In addition, the reaclion rate and amount reacted were calculated for 23 values to time. Thus, Eq. (26) was used entirely as an internal computation device by the computer to facilitate the calculation of the inverse Laplace transform of the rate, other functions derivable from the inverse Laplace transform, and smoothed values of the reaction rate.

The test of the accuracy of the rate measurements was the consistency of the data with the conditions (15) through (23). The raw data consisted of the amount of labeled benzene reacting during a measured time interval. Figures 3 and 4 show for run 12 the average rate during each time interval as horizontal lines and the resulting rate curve calculated from Eq. (26). The average deviation of Eq. (26) from the experimen-

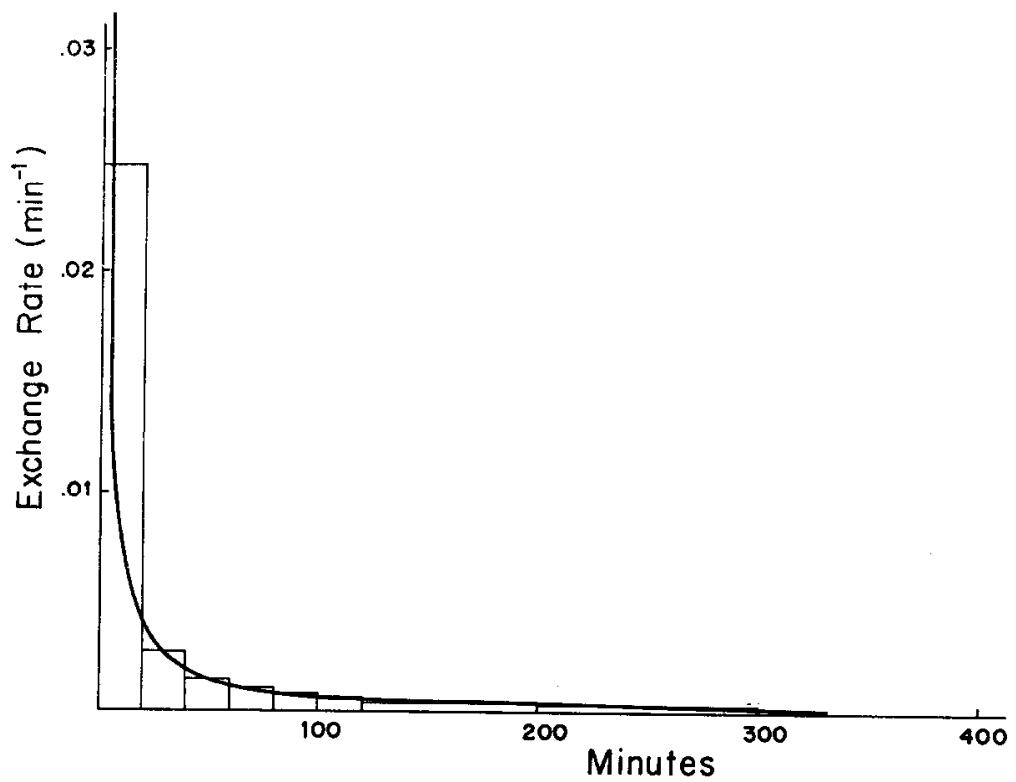

FIG. 3. The kinetic isotherm for the first $400 \mathrm{~min}$ of run 12 at $80^{\circ} \mathrm{C}$. The horizontal lines are the experimentally measured average rates for the indicated time intervals. The curve is obtained from Eq. (26). 
tally measured rates varied from 1.2 to $2.6 \%$. If the deviations were reported in terms of the integrated rate, these figures would be $<1 \%$ for all runs. steps, making a comparison at various temperatures difficult unless the preselected values for the $k_{i}$ 's are identical with the true rate constants. However, a smoothed curve

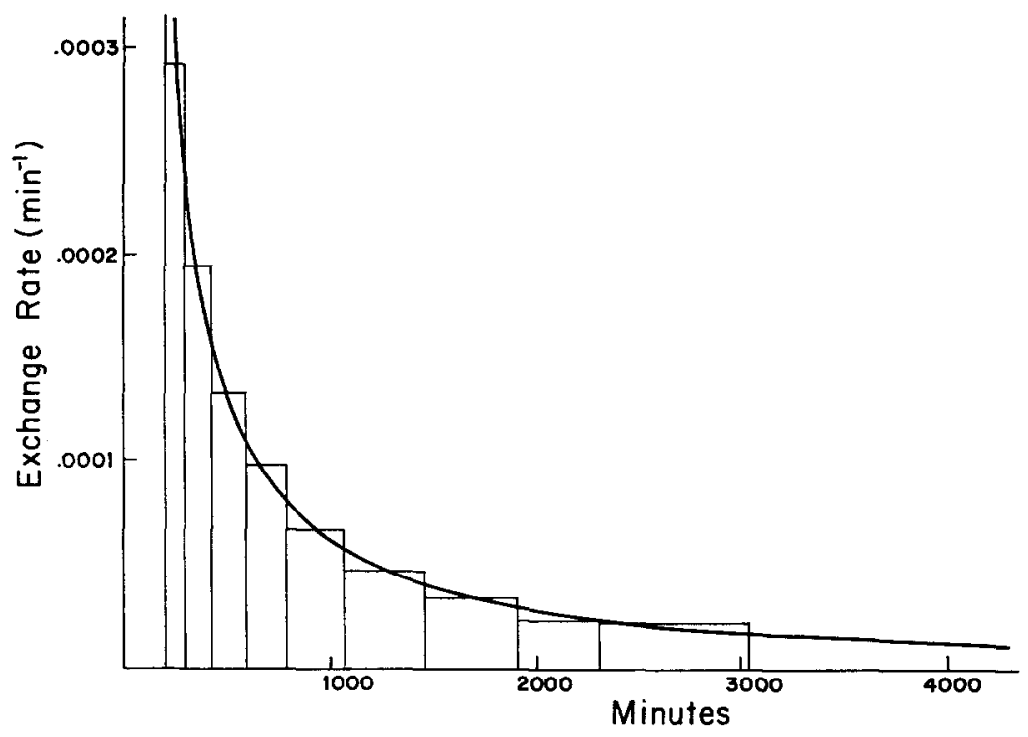

FIG. 4. The kinetic isotherm after 250 min for run 12 at $80^{\circ}$.

Another equation for the correlation of the rate data deserves mention. If one assumes that the surface is composed of a small number of homogeneous regions, then the rate could be expressed by:

$$
r(t)=\sum_{i=1}^{N} a_{i} k_{2} \mathrm{e}^{-k_{i} t}
$$

where $a_{i}$ is the fraction of adsorption sites having rate constant $k_{i}$. Such an equation can be a satisfactory expression of the rate provided a sufficiently large number of terms is used since each adsorption site may be thought of as a homogeneous region. It is quite possible that Eq. (29) could correlate the rate data with about the same accuracy as Eq. (26). The $k_{i}$ 's should be preselected since it is very difficult to make a least squares fit of an exponential function. The resulting distribution function is very simple:

$\left.\begin{array}{l}\rho\left(k_{i}\right)=a_{i} \\ \rho(k)=0 \text { for } k \neq k_{i}\end{array}\right\} i=1,2, \ldots N$

The corresponding integral distribution function, $S(E)$, will appear as a series of of $S(E)$ could be used in order to obtain an approximation of the activation energy distribution.

\section{Calculation of the Activation Einergy Distribution for Reaction (1)}

Equation (28) was used to calculate $\rho(k)$ and $S(E)$ as a function of $k$ for each of the seven runs shown in Table 1 . The coefficients $a_{j}$ and $b_{j}$ for each run were determined by a least squares fit of the rate data of each run to Eq. (26). Under the assumptions made in the derivation of Eq. (12), each value of $S(E)$ corresponds to certain adsorption sites and this correspondence holds at all temperatures. The activation energy corresponding to each value of $S(E)$ is found from a plot of $-\ln k$ versus $1 / T$ at constant $S$. Such a plot for the seven runs is shown in Fig. 5. The slopes of the lines are the values of $E / R$ corresponding to the respective values of $S$. Figure 6 shows a plot of $-T \ln k$ versus $T$ for the same runs and the same $S$ values. The slopes of these lines are $-\ln k_{0}$. The values of $k_{0}$ and $E$ computed from these 


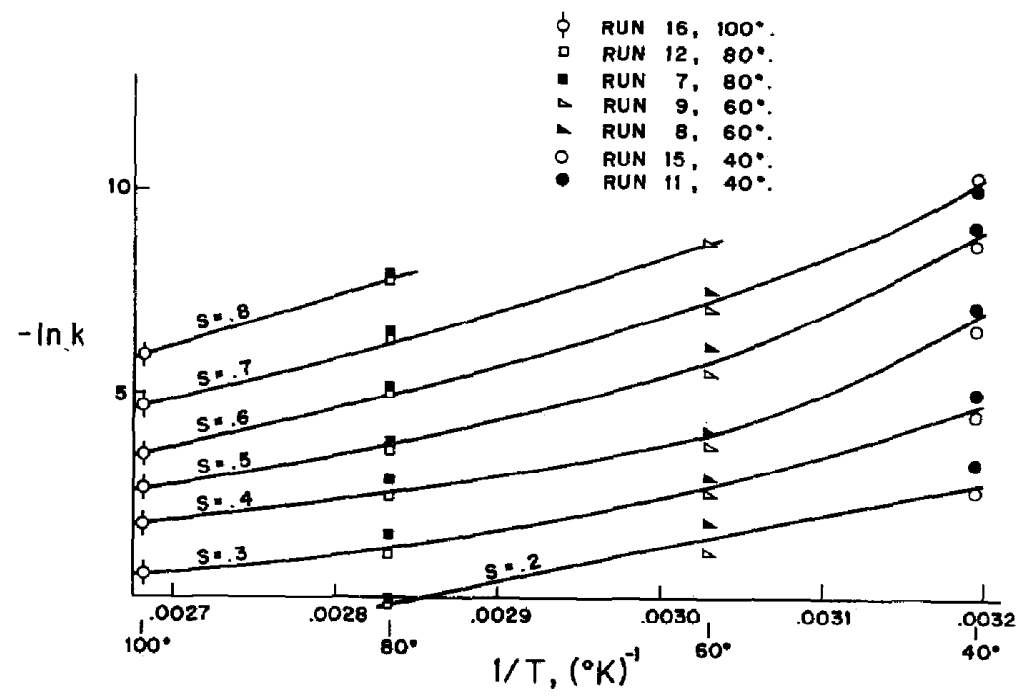

Fra. 5. A plot of $-\ln k$ vs. $1 / T$ at various values of the integral distribution function. The average slope of each line is equal to the corresponding value of $E / R$.

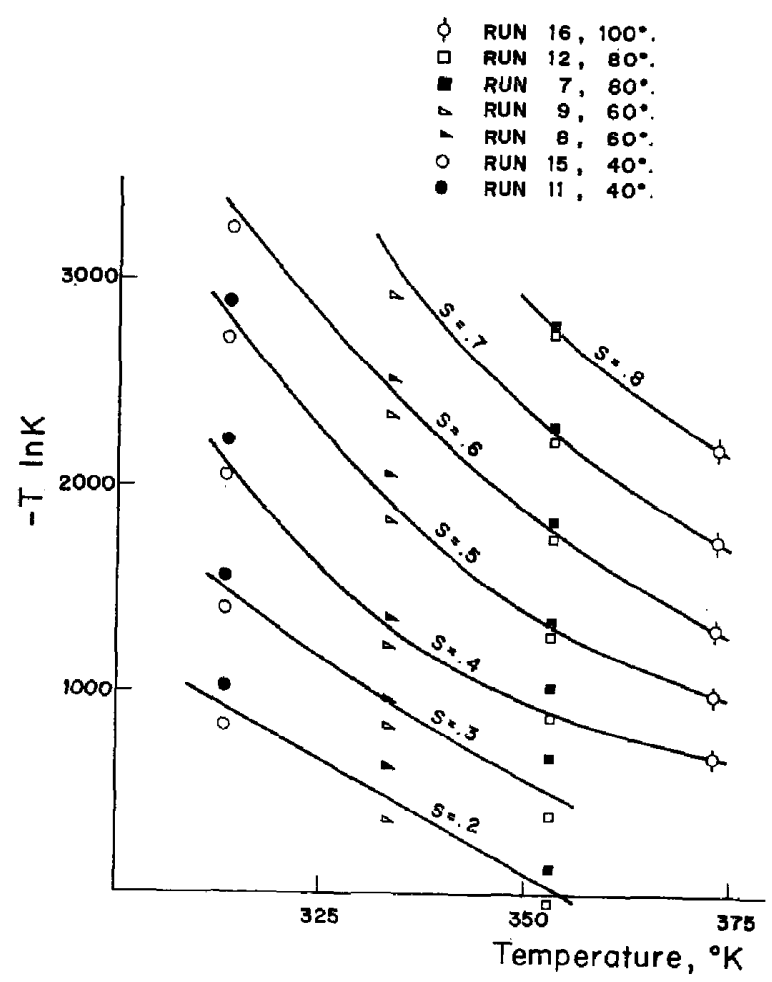

Fig. 6. A plot of $-T$ ln $k$ vs. $T$ at various values of the integral distribution function. The average slope of each line is equal to the corresponding value of $-\ln k_{0}$. 
plots for the various values of $S(E)$ are shown in Table 2. From this table it may be

\section{TABLE 2}

Activation Energy, $E$, and Pre-exponential Factor, $k_{0}$, as Functions of the Integral Distribution Function

\begin{tabular}{ccc}
\hline$S(E)$ & $E$ & $\ln k_{0}$ \\
\hline .20 & 15750 & 22.3 \\
.30 & 18500 & 24.4 \\
.40 & 23000 & 29.4 \\
.50 & 28000 & 37.0 \\
.60 & 30600 & 39.8 \\
.70 & 33700 & $\mathbf{4 4 . 0}$ \\
.80 & 37000 & 46.0 \\
\hline
\end{tabular}

seen that the pre-exponential factor increases with $E$. A plot of $\ln k_{0}$ versus $E$ in Fig. 7
Figure 8 shows a plot of $S(E)$ versus $E$ for the seven runs. A smoothed plot of $S(E)$ is shown in Fig. 9. The activation energy distribution function is shown in Fig. 10. The latter plot is an average for the seven runs. It is roughly estimated that the deviation of a typical run from Fig. 10 is about $25 \%$.

\section{Discussion}

The calculations performed in the previous section can be checked by computing the reaction rates at various temperatures by a numerical integration of the distribution function. The result, obtained with the aid of an IBM 709 digital computer (15), is shown in Fig. 11. The experimentally measured rates are also plotted on the same

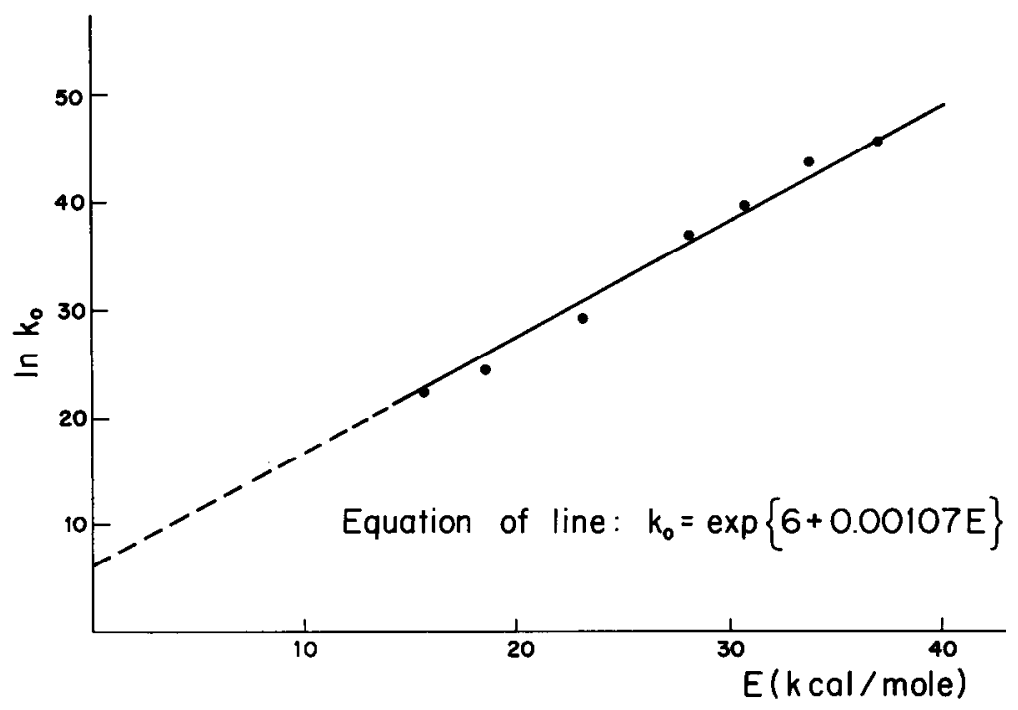

Fig. 7. The dependence of the pre-exponential factor on the activation energy.

shows that the relationship between $k_{0}$ and $E$ is well represented by the equation

$$
k_{0}=\exp (6 .+0.00107 E)
$$

From this equation one obtains the derivative

$$
\beta=d\left(\ln k_{0}\right) / d E=0.00107
$$

which is approximately constant for all values of $E ; \rho(E)$ and $E$ may now be computed for all values of $k$ for each run:

$$
\begin{gathered}
\rho(E)=k \rho(k)(1 / R T-\beta) \\
E=(6 .-\ln k) /(1 / R T-\beta)
\end{gathered}
$$

graph for comparison. At $40^{\circ}, 60^{\circ}$, and $80^{\circ} \mathrm{C}$ the experimental and calculated values show excellent agreement. At $100^{\circ} \mathrm{C}$ good agreement is shown at the longer times (the higher fraction reacted).

In catalytic reactions it is generally assumed that $k_{0}$ is constant at a given temperature. Therefore, it is pertinent to inquire whether there exists some form of the distribution function which, together with a fixed value of $k_{0}$, could produce kinetic isotherms similar to those measured. It would not be ton difficult to find a value of $k_{0}$ and $\rho(E)$ that could reproduce a kinetic 


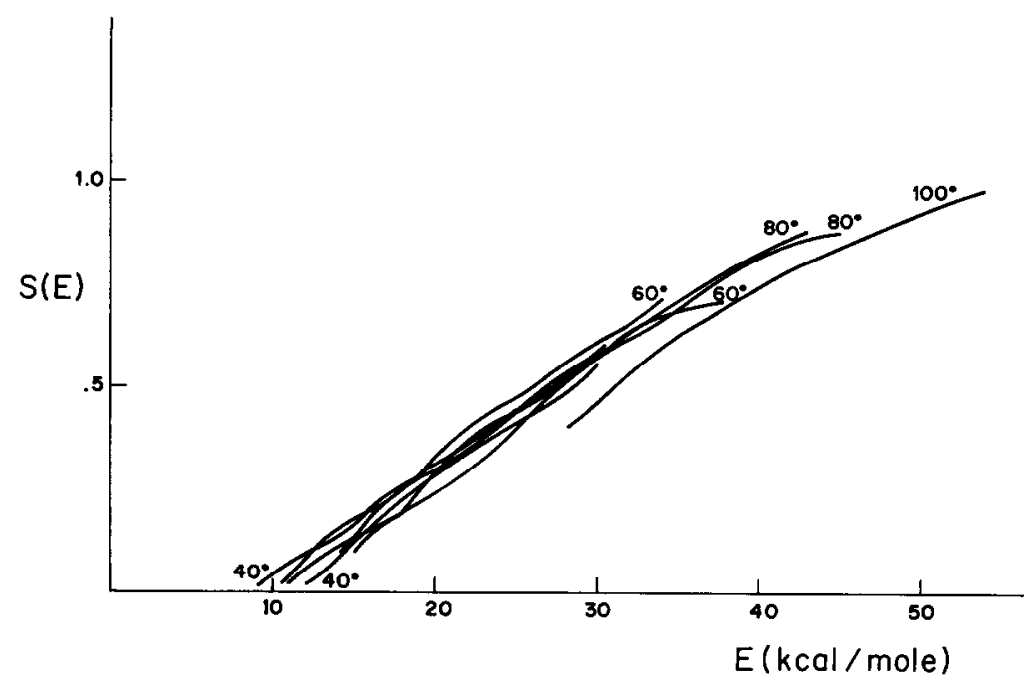

FIG. 8. The integral distribution function calculated for seven runs.

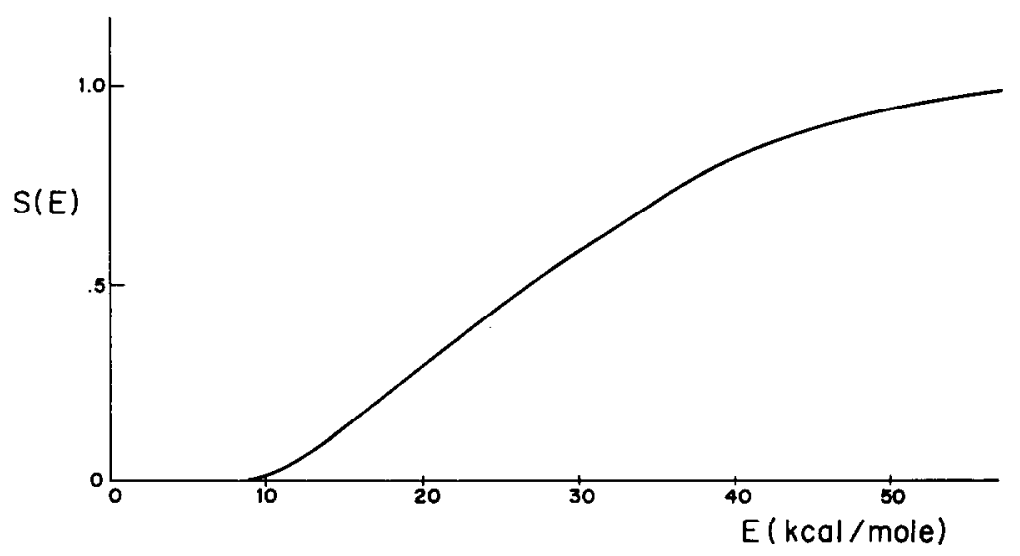

Fig. 9. The integral distribution function for the reaction between gaseous and chemisorbed benzene. Average of seven runs.

isotherm at any one temperature over a rather narrow range of values of $E$. However, for a given value of $E$, the temperature effect is independent of $k_{0}$, and if the predicted rates were in good agreement with the experimental rates at one temperature, the predicted rates at a higher tempcrature would be too high at the start of the reaction (due to a high $k_{0}$ ) and too low after a substantial portion of the labeled specie had reacted (due to a low $k_{0}$ ).

As a further check on the accuracy of the derived distribution of $E$ it was observed that if after about 3 days of reaction at a temperature of $40^{\circ}$ the reactor was heated to $80^{\circ}$ an unusually high increase in reaction rate resulted, indicative of a high $E$. Prior to and during the period of increasing temperature the reactor was purged with helium in order to remove the gas phase benzene and thus stop the reaction. For example, after $4500 \mathrm{~min}$ of reaction (run 11 ) at $40^{\circ} \mathrm{C}$, during which $52 \%$ of the labeled benzene reacted, the temperature was increased to $80^{\circ}$. The reaction rate showed a 230 -fold increase, indicative of an activation energy of about $29.8 \mathrm{kcal} / \mathrm{mole}$. This value is in agreement with the calculated distribution function which shows that $52 \%$ of the adsorbed benzene has an antivation energy 


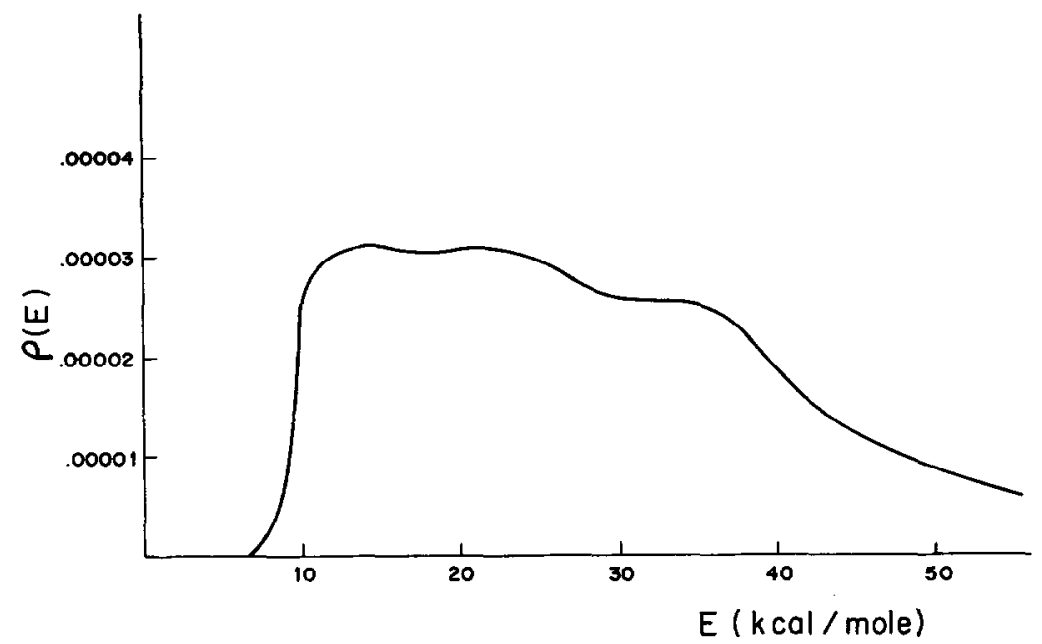

FIG. 10. The activation energy distribution function for the reaction between gaseous and chemisorbed benzene. Average of seven runs.

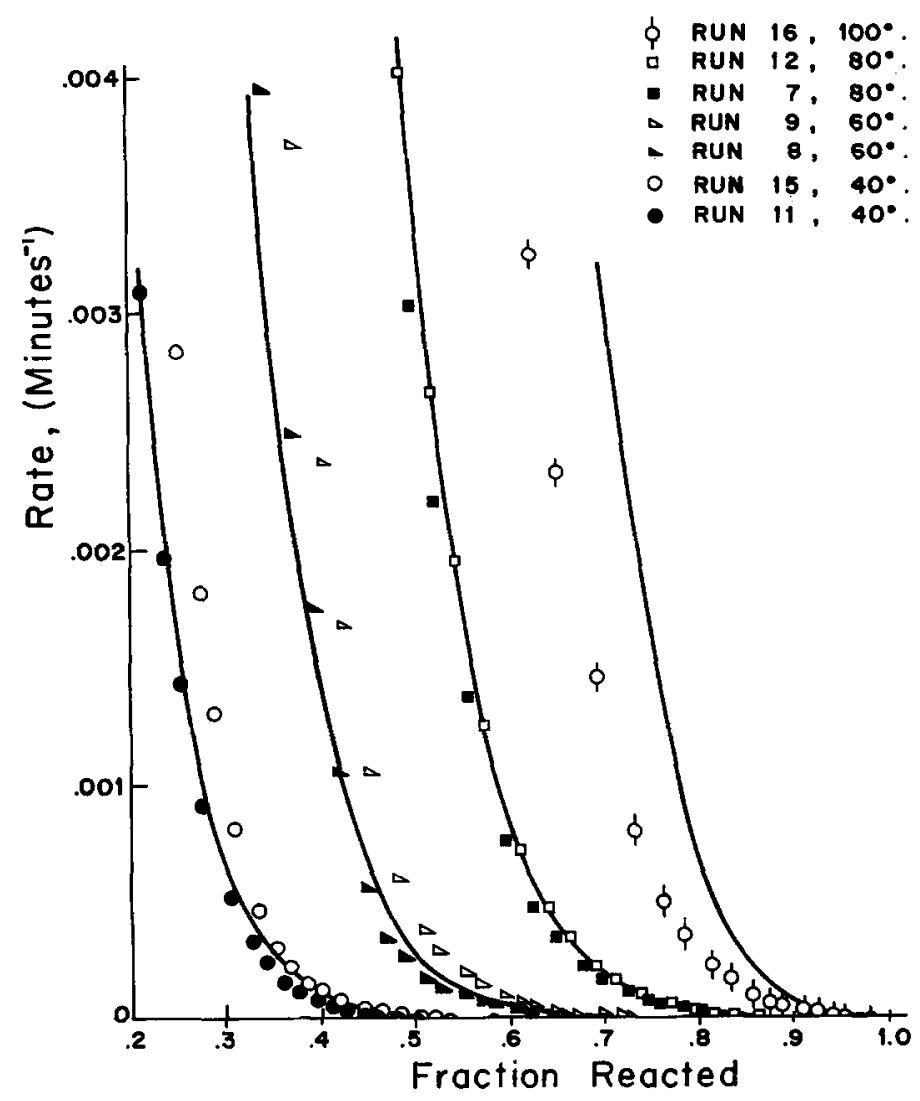

FIG. 11. A comparison of the experimentally measured exchange reaction rates with the rates calculated from a numerical integration of Fig. 9. 
$<28.2 \mathrm{kcal} / \mathrm{mole}$ and the remaining $48 \%$ has an activation energy $<28.2 \mathrm{kcal} / \mathrm{mole}$.

A.ccording to the results of the previous section (Table 2 ), $k_{0}$ varies from site to site with the activation energy ranging from $10^{7}$ to $10^{26} \mathrm{~min}^{-1}$. The usual concept of a pre-exponential factor in catalytic reactions, reflecting the number of active surface sites participating to the reaction, cannot possibly account for this spread of values, especially when one realizes that the rate at which benzene molecules impinge upon a surface is many orders of magnitude lower than the maximum reported value of $k_{0}$.

A frequently observed phenomenon in heterogeneous catalysis is the variation of the activation energy among chemically related catalysts for a given reaction. Ii has been found that catalysts showing high activation energy have a high pre-exponential factor and those showing a low activation energy also have a low pre-exponential factor. This compensation effect is frequently explained on the basis of an exponential type of activation energy distribution in which a high activation energy results in more sites participating in the reaction. The possibility that the compensation effect might have its origin in the active site itself rather than in the distribution or arrangement of active sites has not been given sufficient consideration. This raises the question of the applicability of the Arrhenius equation to all types of surface-gas reactions. An equation of the form

$$
k=\exp \{a-1 \beta-(1 / R T)] E\}
$$

together with a distribution function for the activation energy may have fundamental significance for surface-gas reactions. However, the validity of the above equation at higher temperatures, where the temperature approaches the value of $1 / R \beta$, is questionable.

The most significant result emerging from the calculated distribution for the activation energies of reaction (1) (Fig. 11) is the fact that within the range 10 to $30 \mathrm{kcal} / \mathrm{mole}$ the distribution function is approximately constant. Thus, it is pertinent to inquire about the physical significance of a catalytic surface showing a "uniform heterogeneity" of its energy content. The distribution of the activation energy among surface sites is related to the statistical fluctuation of the energy in and on the lattice of the solid catalyst under reaction conditions. Essentially, it is dependent upon the manner in which the energy in excess of the value corresponding to the thermodynamic equilibrium of the system is stored among surface sites. The problem of the interpretation of the energy distribution can be discussed from a statistical or a topographical point of view (1). In the latter instance, surface defects (point defects, dislocations, line tensions, grain boundaries, etc.) provide a convenient basis for theoretical interpretations, since the force field of these entities can be useful to characterize the nature of the active surface. Let us assume that the force field around a surface defect falls off according to $z / l^{n} f^{\prime}(m)$ where $z$ and $n$ are constants, $l$ is a characteristic dimension of a surface active region, and $f(m)$ is a function of the amount of atoms adsorbed, $m$. Let us further assume that the activation energy is inversely proportional to the valuc of the excess energy present around the surface site. At constant surface coverage, the variation of the activation energy along $l$ is given by:

$$
E=\left(l_{1} / l\right)^{n} E_{1}
$$

where $E_{1}$ is the value of $E$ at $l=l_{1}$. The distribution of active centers in the active region, $\rho(l)$, can be taken as: $\rho(l)=h m$ where $h$ is an appropriate constant dependent upon the geometry of the area (1). Since $\rho(E) d E=\rho(l) d l$, from (33):

$$
\rho\left(E^{\prime}\right)=h l m\left(E / E_{1}\right)^{1 /(n-1)}
$$

For $n=1$

$$
\rho(E)=\text { constant }
$$

Therefore the "uniform heterogeneity" which has been found in this study can be formally interpreted on the basis of a surface force field $\propto\left(z / l^{n}\right)$ with $n=1$. A discussion of the atomistic details of the heterogeneity is much more difficult, because it involves a knowledge of the mechanism of reaction (1). Since surface sites with $E<10 \mathrm{kcal} /$ mole have not played a significant role in 
the exchange reaction studied, adsorption processes could not possibly have had a significant role as kinetically controlling steps. Probably the surface interaction between benzene and $\mathrm{Pt}$ was a more important contributing factor. There is evidence that metals with vacant inner $d$ shell orbitals can interact with the $\pi$ electrons of a double bond to form $\pi$-bonded complexes (16), which have been used to explain the mechanism of several hydrocarbon reactions $(17)$.

Electrons in $\pi$ bonds in benzene exist initially in the field of 6 carbon atoms but the system can achieve greater stabilization if the number of atoms involved is increased as a result of chemisorption. Thus, assuming that each surface atom contributes one electron to the chemisorption bond, there will be initially 12 pairs of neighboring atoms and 24 electrons to be considered. The bonding will extend over $24 / 12=2$ atom cores, and it will be exactly localized. If, however, each adsorption site contributes more than one electron, the bond will extend over $>2$ atom cores and delocalization will set in. In this view, surface heterogeneity will be the result of different electronic states within the same surface site. The process of delocalization requires an activation energy, since electrons must be supplied from the Brillouin zone of the metal. Thus, a slow activated change of the electronic state of some adsorption sites could be invoked for the interpretation of the reported 'aging effects of the benzene.

\section{Conclusions}

1. It has been demonstrated that the rates of isotopic exchange between gaseous and chemisorbed molecules can provide a means of determining the distribution of activation energies with respect to this reaction. The mathematical procedure was discussed and a technique was developed for calculating the activation energy distribution from the kinetic isotherms.

2 . For the reaction between gaseous benzene and benzene chemisorbed on platinum black the reaction rates were measured at $40^{\circ}, 60^{\circ}, 80^{\circ}$, and $100^{\circ} \mathrm{C}$ and from these measurements the activation energy distribution was calculated. The range of acti- vation energies for this reaction was found to be from below 10,000 to beyond 50,000 $\mathrm{cal} / \mathrm{mole}$.

3. It has been shown that for this reaction the pre-exponential factor in the Arrhenius equation is not a constant, as is usually assumed, but varies with the activation energy according to the relation

$$
k_{0}=\exp (6 .+0.00107 E)
$$

The range of pre-exponential factors was found to be from about $10^{7}$ to $10^{26} \mathrm{~min}^{-1}$. Because of this the applicability of the Arrhenius equation to surface-gas reactions is questioned.

4. As a check on the activation energy distribution and the above relationship for the pre-exponential factor, the kinetic isotherms at several different temperatures were calculated by a numerical integration of the activation energy distribution. 'These calculated rates showed good agreement with the experimentally measured rates.

\section{ACKNOWLEDGMENT}

This research was supported in part by a grant from the Esso Research and Engineering Company. We wish to express our gratitude for this support.

\section{REFERENCES}

1a. Roginsky, S. Z., "Adsorption und Katalyse an Inhomogenen Oberflachen. Akademie-Verlag, Berlin, 1958. (First published in USSR in 1948.)

1b. Levin, V. I., Problemy Kinetiki i Kataliza Akad Nauk S.S.S.R. 7, 297-335 (1949).

2. Eischens, R. P., J. Am. Chem. Soc. 74, 61676171 (1952).

3. Roginsky, S. Z., Problemy Kinetiki i Kataliza Akad Nauk S.S.S.R. 9, 5-30 (1957); Zhur. Fiz. Khim. 32, 737-745 (1958).

4. Schuit, G. C. A., deBoer, N. H., Dorgelo, G. J. H., ANd Van ReiJn, L. L., in "Chemisorption" (W. E. Garner, ed.), p. 39. Academic Press, New York, 1957.

5. Suhrmany, R., Heyne, H. J., Weduer, G., $J$. Catalysis 1, 208 (1962).

6. Boreskov, G. K., Vassilevitch, A. A., in "Actes du Deuxieme Congress International de Catalyse, p. 1095. Editions Technip, Paris, 1961.

7. Selwood, P. W., J. Am. Chem. Soc. 79, 4637 (1957); and in "Actes du Deuxieme Congress International de Catalyse," pp. 18101811. Éditions Technip, Paris, 1961. 
8. Pitkethly, R. C., and Goble, A. G., in "Actes du Deuxieme Congress Intermational de Catalyse," pp. 1851-1859. Éditions Technip, Paris, 1961.

9. For a review see "Catalysis" by G. C. Bond. Academic Press, New York, 1962.

10. Brenner, J. G. M., Disc. Faraday Soc., p. 79 (1950).

11. Suhrmann, R., Adv. in Catalysis 7, 303-352 (1955).

12. Gryaznov, V. M., Sheemolis, V. 1., and YAGoDovsky, V. D., Doklady Akad. Nauk SSSR 132, 1132-1135 (1960).
13. Churchill, R. V., "Operational Mathematics," p. 183, 2ud ed. MeGraw-Hill, New York, 1958.

14a. Roginsky, S. Z., Acta Phys. Chim. 20, 227 (1945).

1/4b. Levin, V. I., Problemy Kinetiki $i$ Kataliza Akad Nauk S.S.S.R. 7, 279-335 (1949).

15. For further details see: Brundege, J. A., Ph.D. Thesis, University of Michigan, 1962.

16. Fischer, E. O., Intern. Conf. Coordination Chemistry, Chemical Society (London) p. 73 (1959).

17. Rooney, J. J., J. Catalysis 2, 53 (1963). 